\section{Un nuevo caso de acidosis láctica por buformina}

\section{Sr. Director:}

Las biguanidas se utilizan para el tratamiento de la diabetes desde mediados del siglo XX.

Actualmente están comercializadas dos en España: metformina y buformina, tras la retirada de femformina por los casos de acidosis láctica graves $(1,3)$.

El uso de la metformina dentro de sus indicaciones estrictas es seguro y avalado por múltiples estudios, acaparando la mayoria de las prescripciones de este tipo de antidiabético oral. (2)

Consideramos interesante comunicar un nuevo caso de acidosis láctica por buformina ("Silubin Retard") y recordar las contraindicaciones de uso $(1,4)$.

Paciente mujer de 89 años de edad con antecedentes personales de diabetes tipo 2 tratada con glibenclamida y buformina desde hace 10 años, enfermedad de Alhzeimer moderada, HTA esencial e infecciones urinarias de repetición; remitida por su Médico de Familia al Servicio de Urgencias de un gran Hospital de Madrid por descompensación de su diabetes y mal estado general, fue valorada recomendándose aumentar la dosis de Silubin Retard (pasar de $1 / 2 \mathrm{cp}$ cada 12 horas a $1 \mathrm{cp}$ cada 8 horas); desde entonces en su domicilio presenta mayor deterioro del estado general, dolor abdominal difuso, desorientación y por último disminución del nivel de conciencia.

Dado el progresivo deterioro su familia decide trasladarla a su ciudad natal acudiendo al Servicio de Urgencias de este hospital. A la exploración física presentaba mal estado general, TA 100/50 y obnubilación.

Analíticamente presentaba: glucosa 563, urea 196, Cr 3.34, Ph 7.1, PCO2 22, PO2 100, HCO3 9, E.B -16, Sat O2 98\%, anión GAP 40 y lactato sanguíneo de $40 \mathrm{mg} / \mathrm{dl}$.

Con el diagnóstico de sospecha de acidosis láctica por buformina se ingresó a la paciente tratandose con bicarbonato sódico y sueroterapia, con inicial mejoria y normalización de la acidosis, pero posteriormente gran deterioro en las siguientes 24 horas con reaparición de la acidosis a pesar del tratamiento.

Se indicó a la familia la probable necesidad de hemodiálisis por el severo deterioro de la función renal decidiéndose tratamiento conservador por la situación basal de la paciente con mala evolución hasta el fallecimiento.

La acidosis láctica por biguanidas se debe al aumento de la glucolisis anaerobia, inhibición de la neoglucogénesis hepática y al aumento de la producción intestinal de lactato.

El factor predisponente más importante es la insuficiencia renal que dificulta la eliminación y favorece su acúmulo.

La clínica predominante es dolor abdominal difuso, obnubilación y taquipnea.

El diagnóstico se establece en presencia de acidosis metabólica con anión GAP aumentado y acido láctico aumentado en ausencia de hipoperfusión e hipoxia.

El tratamiento consiste en corrección de la acidosis con bicarbonato y hemodialisis cuando exista importante deterioro de la función renal $(5,6)$.

La mortalidad aproximada es del $50 \%$ de los casos.

En este caso la acidosis se presentó por el aumento de la dosis de la biguanida asociada a un probable deterioro de la función renal multifactorial (¿deshidratación, encamamiento, insuficiencia renal previa?)

Nos parece relevante recordar que en España se comercializan dos tipos de biguanidas (metformina y buformina) así como que estan contraindicadas en presencia de insuficiencia renal, hepática y cardiaca.

\section{A. Martínez Laso}

Servicio de Medicina Interna. Hospital Ciudad de Coria. Cáceres

1. Montero Alonso M, Salvador Cervello G, Perello Roso A, Roig Osca MA. Acidosis láctica asociada al uso de buformina. Rev Clin Esp 2003; 203: 216.

2. Solano Remirez M, González Arencibia C, Álvarez Frías M, Llorente B, Echegaray Agara M. Acidosis láctica en paciente diabético tratado con metformina. An Med Interna (Madrid) 2004 ; 21: 288-290.

3. Rojas Hidalgo E. Biguanidas antidiabéticas y acidosis láctica. Rev Clin Esp 1997; 197: 568-73.

4. Blasco Navaltropo MA, Zaragoza Crespo R, Olivares Toledo D, Alaman Laguarda G. Acidosis láctica y buformina. Rev Clin Esp 1996; $196: 880$.

5. Pena JM, Pernaute R, Vicente C. Acute kidney failure and severe lactic acidosis caused by metformin successfully treated with hemodialysis. Nefrologia 2004; 24: 89-90.

6. Panzer U, Kluge S, Kreymann G, Wolf G. Combination of intermittent haemodialysis and high-volume continuous haemofiltration for the treatment of severe metformin-induced lactic acidosis. Nephrol Dial Transplant $2004 ; 19: 2157-8$. 
Diplegia facial periférica como manifestación inicial de un síndrome de Guillain-Barré secundario a una infección aguda por citomegalovirus

\section{Sr. Director:}

La afectación del nervio facial en el síndrome de GuillainBarré (SGB) es frecuente en el curso de la enfermedad (entre un $20-60 \%$ casos) pero la afectación bilateral periférica, aun siendo característica, es poco frecuente $(<1 \%$ de los casos) y todavía menos si ésta es la manifestación inicial del síndrome (1-3)

A continuación presentamos un caso de un SGB que se manifestó inicialmente como una diplegia facial periférica en una paciente con el antecedente de una infección viral del tracto respiratorio superior en las dos semanas previas al ingreso.

Se trata de una mujer de 36 años, ganadera de profesión, que consultó en urgencias por desviación de la comisura bucal y parestesias en la punta de los dedos de ambas manos.

Como antecedentes presentaba una migraña menstrual, era fumadora de 20 cigarrillos al día y no refería otros hábitos tóxicos.

Unos 20 días antes de la consulta en urgencias, la paciente presentó un catarro común con artromialgias y sensación distérmica. Las parestesias en las manos se iniciaron 7 días después y fueron progresivamente aumentando de intensidad hasta el día de la consulta en que se añadió desviación de la comisura bucal a la derecha con imposibilidad para la oclusión del párpado izquierdo y disestesias en la hemicara izquierda. En la exploración destacaba unas adenopatías cervicales, pérdida de fuerza en las extremidades inferiores con hipo-arreflexia y una parálisis facial bilateral periférica. La sensibilidad era normal. En la analítica destacaba una GPT 61 U/l, GOT $32 \mathrm{U} / \mathrm{I}$, VSG $19 \mathrm{~mm} / \mathrm{h}$ y un hemograma normal con linfocitos atípicos (> 10\%) en el frotis. Las pruebas de imagen (tomografía axial computerizada y resonancia magnética) mostraron un quiste aracnoideo como única alteración. Las serologías para Borrelia, Ricketsias, Chlamidea psitacci y pneumoniae, Brucella, Salmonella, Coxiella, Mycoplasma y lúes fueron negativas, presentando inmunidad frente al virus de la hepatits B y frente al virus de Ebstein-Barr. El HIV fue negativo. Obtuvimos una serología IgM positiva para citomegalovirus en suero repetida en dos ocasiones compatible con infección aguda. El resto del estudio analítico incluidos marcadores tumorales, hormonas tiroideas, inmunofijación, copro y uroporfirinas, ANCA, enzima convertidora de la angiotensina (ECA) y factor reumatoide resultaron negativos.

Se realizó una punción lumbar en la que objetivamos un líquido claro sin leucocitos ni hematíes, con una glucosa normal (1:1 con el suero) y unas proteínas de $286 \mathrm{mg} / \mathrm{dl}$ (albúmina $78 \mathrm{mg} / \mathrm{dl}$ ). Los antígenos bacterianos, la citología para células malignas, el ADA, la ECA, los cultivos -incluidos para tuberculosis-y las serologías así como la reacción en cadena de la polimerasa (PCR) para virus herpes fueron negativas un mes después de haberse iniciado la clínica.

El electromiograma mostró una polirradiculoneuropatía sensitivomotora de predominio axonal con discretos signos de denervación activa en extremidad superior derecha y más marcado en extremidades inferiores.

Con el diagnóstico de síndrome de Guillain-Barré, según los criterios de Asbury (Tabla I) en su forma de diplegia facial, parestesias e hipo-arreflexia secundario a un síndrome mononucleósido por CMV, se inició tratamiento con inmunoglobulinas intravenosas presentando una recuperación ad integrum en 5 semanas. La serología IgM para CMV se negativizó sin tratamiento ya que la paciente era inmunocompetente.

La diplejia facial representa menos del $2 \%$ de todas las parálisis faciales periféricas y se define como aquella que afecta a los dos lados de la cara en un plazo de tiempo no superior a las 4 semanas (2). En este sentido, la afectación del nervio facial es frecuente en el SGB (20-60\% de los casos en el curso de la enfer-
TABLA I

CRITERIOS DIAGNÓSTICOS DEL SÍNDROME DE GUILLAIN BARRÉ (CRITERIOS DE ASBURY 1981) $(1,5)$

Características requeridas para el diagnóstico

Tetraparesia progresiva

Arreflexia

Características que sustentan el diagnóstico

Progresión rápida de los síntomas (hasta 4 semanas)

Relativa simetría de los síntomas y signos

Compromiso de pares craneales sobretodo el VII

Síntomas y signos sensitivos leves

Recuperación que se inicia entre 2 y 4 semanas después de finalizada la progresión

Disfunción autonómica

Ausencia de fiebre al inicio de los síntomas

Características de laboratorio que sustentan fuertemente el diagnóstico

Hiperproteinorraquia

Mononucleares en el LCR (> $10 \mathrm{c} / \mathrm{ml}$ ) después de una semana

Estudio electrofisiológico compatible con desmielinización

Exclusión de otros trastornos que causan parálisis flácida aguda

medad) pero, aunque es característica, la forma de presentación como una diplegia facial periférica es poco frecuente $(<1 \%)$ y típicamente son los cuadros secundarios a la infección por CMV los que cursan de este modo $(2,3)$. Las etiologías más frecuentes de esta afectación bilateral son las causas benignas- alrededor de un 50\% de las mismas-(parálisis bilateral idiopática de Bell (23\% del total), Guillain-Barré (10\% del total), neuropatías craneales múltiples, esclerosis multiple,enfermedad de Parkinson) seguidas de los tumores meníngeos y de tronco $(21 \%)$ y de las infecciones por borrelia, sífilis, HIV, virus herpes, mononucleosis, etc. $(11 \%)$. Otras causas pueden ser las congénitas (Sd. Moebius, miopatías), porfiria, sarcoidosis, amiloidosis, leucemias, linfomas, diabetes, ingesta de etilenglicol, alcoholismo $(3,4,6)$.

El manejo de esta parálisis facial bilateral pasa por el tratamiento etiológico que la causa no debiendo instaurar tratamiento empírico con corticoides ya que pueden estar contraindicados en los procesos subyacentes; en nuestro caso el tratamiento con inmunoglobulinas hizo desaparecer la sintomatología.

En conclusión, una parálisis facial bilateral periférica con o sin afectación sensitivomotora obliga a considerar el SGB entre los diagnósticos diferenciales a realizar (6).

\section{P. J. Marchena Yglesias, E. Val Pérez, L. P. de Benito Cor- dón, M. J. Hervás Laguna}

Servicio de Medicina Interna. Hospital Virgen de la Luz. Cuenca

1. Mellado P, Sandoval P. Variantes regionales del Síndrome de GuillainBarré. Cuadernos de Neurología: Publicaciones de la Universidad Católica de Chile 2002; vol 26.

2. García Callejo, et al. Parálisis facial bilateral periférica y síndrome de Guillain-Barré. Acta Otorrinolaringol Esp 1998; 49 (7): 561-8.

3. Keane JR. Bilateral seventh nerve palsy: analisis of 43 cases and review of the literatura. Neurology 1994; 44 (7): 1198-202.

4. Morales Angulo et al. Paralisis facial bilateral simultanea. Acta Otorrinolaringol Esp 1995; 46 (4): 305-309.

5. Asbury AK, Arnason B, et al. Criteria for diagnosis of Guillain-Barré syndrome. Ann Neurol 1978; 3: 565-6.

6. González Santiago MP, et al. Parálisis facial bilateral como forma de presentación de síndrome de Guillain-Barré. An Pediatr (Barc) 2003; 58 (1): 77. 


\section{Un nuevo caso de neurosífilis latente tardía de presentación atípica}

Sr. Director:

La afectación del sistema nervioso central por Treponema pallidum se produce en el 5-10\% de los infectados y hasta en la tercera parte de los casos que progresan hasta estadios avanzados se desarrolla la neurosífilis $(1,2)$.

Actualmente predominan las formas de presentación atípica, respecto a las formas clásicas de parálisis general progresiva y tabes dorsal (3).

Recientemente hemos tenido ocasión de diagnosticar un nuevo caso de presentación atípica y nos parece interesante comunicarlo brevemente.

Mujer de 67 años de edad, con antecedentes personales de intervención de prótesis de rodilla izquierda hacía un año, estenosis pilórica secundaria a ulcus, esofagitis distal, en tratamiento con omeprazol.

Síndrome ansioso - depresivo en seguimiento desde hacía 4 años por psiquiatría, en tratamiento con clorazepato dipotásico, clorpromazina y amitriptilina.

Por cambio de domicilio, acudió a nuestra área sanitaria, el psiquiatra solicitó una serología a sífilis (RPR), que fue positiva, remitiéndola a nuestras consultas.

La exploración física reveló una paciente con deterioro cognitivo leve, sin datos de focalidad neurológica, marcha normal, pupilas isocóricas y normorreactivas. Auscultación cardiaca y pulmonar normales. Abdomen: blando, no doloroso, no organomegalias.

Laboratorio: hemograma, coagulación y bioquímica normales.

Radiografía de tórax: elongación aórtica, no cardiomegalia, campos pulmonares claros.

Anticuerpos antitreponema pallidum en el suero positivo a título de $1 / 640$.

La TAC craneal, mostró atrofia cerebral y un pequeño infarto lacunar insular derecho.

Se realizó una punción lumbar, obteniéndose un líquido claro, con 3 leucocitos mononucleares, $45 \mathrm{mg} / \mathrm{dl}$ de proteínas y 80 $\mathrm{mg} / \mathrm{dl}$ de glucosa. Se detectaron en el líquido cefalorraquídeo anticuerpos IgG antitreponema pallidum por enzimoinmunoanálisis (EIA) positivos a título 1.85, siendo el título de anticuerpos IgM negativos.

Se diagnóstico neurosífilis tardía latente y recibió tratamiento con penicilina sódica a dosis de 4 millones de unidades / 4 horas, durante 10 días.

Se observó una evolución francamente favorable del cuadro psiquiátrico, habiendo reducido de un modo drástico la medicación.

Estamos pendientes de una próxima analítica de líquido cefalorraquídeo y de nuevos estudios serológicos en el mismo.

La neurosífilis puede presentarse en la forma de cuadros psiquiátricos; los más frecuentes son cuadros psicóticos con alucinaciones auditivas e ideas paranoides, también pueden observarse cuadros delirantes, síntomas conversivos y alteraciones de la personalidad que pueden ser algunas de las manifestaciones psiquiátricas de la neurosífilis (4).

En las formas atípicas de la neurosífilis la TAC y la RNM cerebral pueden ser normales o mostrar un amplio espectro de anormalidades como atrofia corticosubcortical, infartos cerebrales, lesiones inespecíficas en la sustancia blanca cerebral , captación meníngea, datos de arteritis, lesiones de goma sifilítica, etc (2).

Es necesario estudiar el líquido cefalorraquídeo (LCR) cada 3 a 6 meses durante unos 3 años, después del tratamiento de casos sintomáticos o asintomáticos de neurosífilis o bien hasta que los parámetros del líquido cefalorraquídeo retornen a la normalidad.

El título de reagina en el LCR desciende gradualmente en el período de varios años. Se sugiere que los parámetros séricos y del LCR se pueden normalizar más lentamente en los pacientes infectados por el virus de la inmunodeficiencia humana y con neurosífilis, en comparación con las personas no infectadas por el VIH (5).

La administración de bencilpenicilina intravenosa a dosis de 12 millones de unidades o más al día, durante unos 10 días, parece garantizar la aparición de concentraciones treponemicidas de bencilpenicilina en el LCR (5).

\section{F. Marcos Sánchez, I. Albo Castaño, F. Árbol Linde, J. Colás Rubio}

Servicio de Medicina Interna. Hospital Nuestra Señora del Prado. Talavera de la Reina. Toledo

1. Holland BA, Perrett LV, Mills CM. Meningovascular syphilis: CT and MR findings. Radiology 1986; 158: 439-442.

2. Brighbill TC, Ihmeidan IH, Post MJ, Berger JR, Katz DA. Neurosyphilis in HIV-positive and HIV-negative patients: neuroimaging findings. Am J Neuroradiol 1995; 16: 303-311.

3. Harris DE, Enterline DS, Tien RD. Neurosyphilis in patients with AIDS Neuroimaging Clin N Am 1997; 7: 215-221.

4. Rundell JR, Wise MG. Neurosyphilis: a psychiatric perspective. Psychosomatics 1995; 26: 287-295.

5. Lukehart SA, Holmes KK. Enfermedades producidas por espiroquetas. Sífilis. En: Principios de Medicina Interna de Harrison. 14 edición. McGraw-Hill-Interamericana. Madrid 1998; 1172-1184.

\section{Hipertensión portal por cavernomatosis esplenoportal}

\section{Sr. Director:}

La causa más frecuente de hipertensión portal en nuestro medio es, con mucho, la cirrosis hepática $(1,2)$. Sin embargo, la hipertensión portal puede ser también, aunque mucho menos habitualmente, de tipo postsinusoidal o presinusoidal (prehepática o intrahepática).

Presentamos el caso de un varón de 39 años que ingresa en nuestro centro por un episodio de vómitos cuantiosos de sangre fresca con anemización importante, objetivándose la endoscopia digestiva alta varices esofágicas (II/IV), fúndicas y bulbares con gastropatía hipertensiva y restos hemáticos oscuros. El estudio etiológico de hepatopatía crónica (incluyendo serologías para virus hepatotropos, porfirinas, cobre, ceruloplasmina, ferritina y alfa-1-antitripsina) es normal y las pruebas de imagen abdominal (que incluyen arteriografía mesentérica con estudio de retornos venosos y esplenoportografía [Fig. 1]) objetivan la existencia de hipertensión portal prehepática por cavernomatosis portal con abundantes derivaciones venosas perivesiculares y subhepáticas, con trombosis de la vena esplénica y gran esplenomegalia. La biopsia hepática transyugular evidencia una histología hepática normal. En el estudio de hipercoagulabilidad presenta déficit de proteína S. Valorado por Servicio de Cirugía, se desestima intervención derivativa portosistémica por las escasas posibilidades técnicas del eje esplenoportal y el dudoso beneficio esperable y el paciente es dado de alta con el diagnóstico de hemorragia digestiva alta por gastropatía hipertensiva en el seno de hipertensión portal prehepática por trombosis esplenoportal con 
transformación cavernomatosa probablemente secundaria a estado de hipercoagulabilidad por déficit funcional de proteína S. Evoluciona sin nuevos episodios de sangrado bajo tratamiento con betabloqueantes y antisecretores gástricos. No se plantea, por el momento, anticoagulación al respecto de su probable estado de hipercoagulabilidad, considerando el potencial riesgo de sangrado digestivo de su patología esplenoportal, no subsidiaria de tratamiento quirúrgico definitivo.

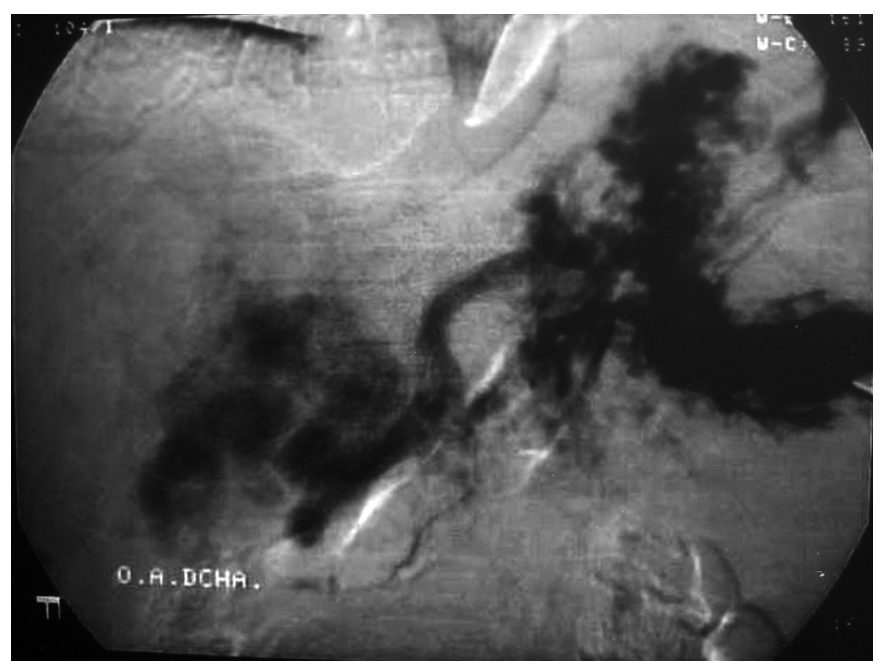

Fig. 1. Imagen de la esplenoportografía del paciente que muestra la mayoría del contraste retenido en bazo, con escaso relleno portal a través de ramas gástricas cortas y gástrica izquierda.

La trombosis esplenoportal es la causa más frecuente de hipertensión portal prehepática (también denominada hipertensión portal no cirrótica) en España y sus principales causas en adultos son la complicación de intervenciones abdominales o procesos infecciosos, inflamatorias o tumorales intraabdominales; los estados de hipercoagulabilidad; o la cirrosis hepática. No obstante, hasta en el $50 \%$ de los casos no se llega a identificar la causa (2,3-6). El trombo puede asentar a cualquier nivel del eje esplenoportal y de su localización dependerá el tipo de circulación colateral derivativa que se desarrolle. Cuando la luz de la vena porta queda recanalizada y sustituida por multitud de pequeños canales tortuosos se habla de transformación cavernomatosa de la porta $(2,4,5)$.

La principal manifestación clínica de la hipertensión portal no cirrótica es, como en el caso descrito, la hemorragia digestiva por varices esofágicas y gástricas prominentes. El diagnóstico se basa en la evaluación endoscópica, la ecografía hepática y la realización de angiografía arterial (retorno venoso) y venosa (esplenoportografía) que confirma el diagnóstico y determina la extensión. Se requiere biopsia hepática para descartar otras causas de hipertensión portal $(2,4,5)$.

La expectativa de vida de los pacientes con trombosis portal extrahepática está determinada por la causa subyacente que origina la obstrucción de la vena porta. La supervivencia actuarial a los 5 años es del $95 \%$ en pacientes tratados con escleroterapia endoscópica. Las muertes se suelen asociar a neoplasias hematológicas subyacentes al cuadro de trombosis portal $(2,4)$.

El manejo de la trombosis del eje esplenoportal es difícil, no siendo posible, por lo general, el tratamiento quirúrgico definitivo. Se debe valorar la reversibilidad de la etiología, el riesgo del tratamiento, la capacidad de supervivencia a la hemorragia y el acceso a asistencia médica urgente para determinar la actitud terapéutica a seguir. Generalmente no suele ser factible la derivación quirúrgica y se requiere de tratamiento farmacológico con betabloqueantes y endoscópico con escleroterapia o ligadura con bandas de las varices esófago-gástricas sangrantes. Si fracasa el tratamiento endoscópico o hay sangrado por varices distales al esófago, está indicado realizar shunt quirúrgico portosistémico para controlar la hemorragia $(6,7)$. Incluso puede ser necesario realizar devascularización esofagogástrica radical (técnica de Suguira modificada) en caso de varices sangrantes secundarias a hipertensión portal extrahepática no puenteable mediante cirugía derivativa, ya que es la única opción ante oclusión de las venas mayores tributarias de la porta, cuando falla el tratamiento endoscópico. Otra opción es la colocación de stent venoso portal $(6,8)$.

\section{J. F. Varona Arche, J. L. Aranda Arcas}

Servicio de Medicina Interna. Hospital Universitario 12 de Octubre. Madrid

1. Kapoor D, Sarin DK. Pathophysiology of portal hypertension. J Gastroenterol Hepatol 2002; 17s: s482-7.

2. García-Pagán JC, Bosch Genover J. Hipertensión portal. Medicine 2000; 8 (11): 543-548.

3. Molina E, Reddy KR. Noncirrhotic portal hypertension. Clin Liver Dis 2001; 5: 769-87

4. Sanyal AJ. Noncirrhotic portal hypertension: Extrahepatic portal vein obstruction. UptoDate 8.3. October 2000.

5. Denniger $\mathrm{MH}$, Chait $\mathrm{Y}$ et al. Causes of portal or hepatic venous thrombosis in adults: The role of multiple concurrent factors. Hepatology 2000; 21: $587-591$

6. Bosch J, Abraldes JG, Groszmann R. Current management of portal hypertension. J Hepatol 2003; 38s: s54-68.

7. Alam $\mathrm{H}$ et al. Portal vein thrombosis in the adult: Surgical implications in an era of dinamic imaging. Am Surg 1997; 63: 681-684.

8. Harville LE et al.Variceal hemorrhage associated with portal vein thrombosis: treatment with a unique portal venous stent. Surgery 1992; 111: 585-588.

Fracaso hepático agudo grave: marcadores pronóstico y trasplante

\section{Sr. Director:}

El fracaso hepático agudo se origina como consecuencia de una necrosis masiva de los hepatocitos, y se manifiesta por la claudicación de las funciones hepáticas en pacientes con hígado previamente sano. La triada característica es la aparición de encefalopatía hepática, ictericia, hipoprotrombinemia (1). Esta patología entraña una elevada mortalidad (60-95\%). Sin embargo la existencia de trasplante hepático ha cambiado el curso de la enfermedad mejorando notablemente la supervivencia, siendo en la actualidad mayor del $80 \%$ (2).

Por estos motivos, realizamos un estudio descriptivo de los pacientes ingresados con fracaso hepático agudo en un Servicio de Medicina Intensiva de un hospital de tercer nivel, analizando su etiología y existencia de criterios precoces de mal pronóstico, así como la indicación de trasplante hepático desde la implantación de dicho programa en nuestro centro (1998).

Las variables estudiadas fueron la edad, el sexo, el lugar de procedencia, tipo de fracaso hepático agudo grave (FHAG) (fulminante, subfulminante, tardío), etiología del mismo, cumplimiento de criterios establecidos por el King's College (K-C) (Tabla I) $(1,3,4)$, tiempo que tardan en desarrollar dichos criterios, momento en el que se incluye como urgencia 0 , tiempo que transcurre desde ésta hasta la realización de trasplante hepático, evolución posterior (necesidad o no de retrasplante hepático, supervivencia y evolución). 


\section{TABLA I}

CRITERIOS DEL KING'S COLLEGE

\begin{tabular}{l} 
A. Fracaso hepático agudo grave por paracetamol \\
$\mathrm{pH}<7,3$ \\
$\mathrm{O}$ \\
INR $>7,7+$ creatinina $>3,5+$ encefalopatía grado III-IV \\
\hline B. Fracaso hepático agudo grave por otras causas \\
INR $>7,7$ \\
o 3 de las siguientes \\
INR $>3,85$ \\
Bilirrubina $>17 \mathrm{mg} / \mathrm{dl}$ \\
Edad $>10$ años $0<$ de 40 años \\
Causa desconocida \\
Secundaria a tóxicos, virus hepatitis NA-NB \\
Intervalo encefalopatía-ictericia $>7$ días
\end{tabular}

Un total de 10 pacientes fueron ingresados en nuestro servicio debido a FHAG, de ellos el $50 \%$ fueron mujeres y el $50 \%$ varones con una media de edad 34 años. Todos ellos procedían de otros centros hospitalarios. El tipo de FHAG fue fulminante en 9 pacientes, y subfulminante en 1. Las etiologías fueron las siguientes: virus hepatitis B (VHB) en 2 de ellos, autoinmune en 2 casos, criptogenética en 1 , drogas de abuso en 2 , debido a ingesta de paracetamol 1, de otros fármacos (anticonceptivos orales) 1 e ingesta de setas 1 . Requirieron trasplante hepático 8 pacientes y retrasplante 3 debido a incompatibilidad de isogrupo en el trasplante inicial.

En el momento de su traslado cumplían una media de 3 criterios $\mathrm{K}-\mathrm{C}$, siendo considerados como urgencia 0 en una media de 6,5 días, y trasplantados en una media de 27 horas posteriores a dicha consideración. Ninguno de ellos falleció, y el $90 \%$ mostró una buena evolución posterior. La supervivencia en la actualidad es del $100 \%$.

El FHAG es de una entidad poco frecuente (representa el $0,07 \%$ de los ingresos en UCI durante el periodo de tiempo estudiado). Tiene lugar en pacientes jóvenes cursa de modo fulminante y etiología variada. Las drogas $(5,6)$, la autoinmune y la originada por el VHB $(2,7)$ son las etiologías más frecuentes en nuestros resultados. Esta última coincide con la bibliografía consultada (5-8), existiendo otras etiologías descritas como es la intoxicación por paracetamol en el Reino Unido (9-10), o la criptogénica (10) y distintos virus de la hepatitis $(2,4,6)$, en el resto de los países. En nuestro medio resulta habitual la originada por la amanita phaloides, excepcional en paises ajenos al ámbito mediterráneo.

Aunque la mayor parte, no todos requirieron trasplante hepático $(2,8-10)$. Debido a la posibilidad de reversibilidad de la función hepática (8) y a los efectos secundarios que acarrea la inmunosupresión crónica, resulta de vital importancia el conocimiento de los indicadores precoces de mal pronóstico para trasplantar únicamente a los pacientes que fallecerían si no recibieran esta terapia $(1,3,4,10)$. Así pues, 8 pacientes fueron trasplantados en una media de 27 horas de su consideración como urgencia 0 , siendo una cifra inferior tanto a la descrita en otras series (EEUU 4 días) (10) como a la que posee el trasplante hepático no urgente en nuestro servicio (96 horas).Los pacientes no subsidiarios de trasplante hepático presentaron una buena evolución posterior, con restitución ad integrum de la función hepática.

De lo expuesto se deriva que es fundamental el reconocimiento precoz del FHAG, siendo indispensable la valoración de los indicadores precoces de mal pronóstico para detectar qué pacientes deben ser trasladados a centros de referencia que cuentan con programa de trasplante hepático.

\section{B. Obón Azuara, P. Luque Gómez, I. Gutiérrez Cía, B. Villa- nueva Anadón}

\section{Servicio de Medicina Intensiva. Hospital Clínico Universitario.} Zaragoza

1. O'Grady JC, Schalm SW, Williams R. Acute liver failure: redefining the syndromes. Lancet 1993; 342: 273-275.

2. Tessier G, Villeneuve E, Villeneuve JP. Etiology and outcome of acute liver failure: experience from a liver trasnplantation centre in Montreal. Can J Gastroenterol 2002; 16: 672-676.

3. O'Grady JC. Alexander GJM, Hayllar KM, Williams R. Criteria for liver trasplantation in fulminant hepatic failure. Gastroenterol 1989; 97 : 439-445.

4. Shakil AO, et al. Acute liver failure: clinical features, outcome analysis, and applicability of prognostic criteria. Liver Transpl 2000; 6: 163-9.

5. Lange Brock N, Berg T, Muller AR, et al. Acute liver failure following the use of ectasy (MDMA). Z. Gastroenterol 2002; 40: 581-586.

6. De Rave S, Tilanus HW, Van der Linden J, et al. The importance of orthotopic liver transplantation in acute hepatic failure. Transpl Intern 2002; 5: 29-33.

7. Schiodt FV, Davern TJ, Shakil AO, et al. Viral hepatitis-related acute liver failure. American J Gastroenterol 2003; 98: 448-453.

8. Gill RQ, Sterling RK. Acute liver failure. J Clin Gastroenterol 2001; 33 191-198.

9. Williams R. Changing clinical patterns in acute liver failure. J Hepatol 2003; 39: 660-661.

10. Lee WM. Acute liver failure in the United States. Semin Liver Dis 2003; $23: 217-226$.

\section{Rotura de una rama de la arteria pulmonar en el laboratorio de hemodinámica}

\section{Sr. Director:}

La rotura de la arteria pulmonar o de alguna de sus ramas durante inserción de catéteres en cavidades derechas es una complicación poco frecuente pero con alta mortalidad.

El tratamiento de la rotura de la arteria pulmonar es actualmente controvertido e individualizado, pudiendo realizarse indistintamente cirugía torácica o embolización por radiología intervensionista en dependencia de la situación hemodinámica del paciente y la disponibilidad del hospital en que nos encontremos.

Sra. F.H.N de 73 años de edad con antecedentes de AcxFa, DLM reumática con EM ligera, IM de moderada a severa, HTP moderada, clase funcional II-III para disnea, histerectomía a los 57 años, artrosis. En tratamiento habitual con anticoagulantes dicumarínicos, Digital y Aldactone.

Ingresa en el hospital el 3/4/02 de forma programada para realizar estudio hemodinámico y valorar cirugía de recambio valvular.

Se realiza el estudio hemodinámico el día 5/4/03 mediante abordaje femoral derecho con catéter balón 7F y catéter pigtail de $6 \mathrm{~F}$ para el cateterismo izquierdo, angiografías coronarias con catéter Judkins $6 \mathrm{~F}$. El resultado del estudio mostraba una dominancia izquierda con lesión única de $90 \%$ en la segunda porción de circunfleja. La ventriculografía por su parte evidenciaba una cavidad ventricular de tamaño ligeramente aumentado con AI dilatada y regurgitación mitral grado IV, válvula mitral moderadamente calcificada.

Durante la cateterización de la arteria pulmonar se produce una hemorragia pulmonar masiva que obliga a suspender el procedimiento, avisando en ese momento a UCI por inestabilidad de la paciente con TA indetectable, por lo que se procede a intubación y medidas de reanimación. 
La fibrobroncoscopia en la sala de hemodinámica evidencia sangrado en bronquio derecho, se administra adrenalina intrabronquial que es ineficaz.

Se realiza arteriografía pulmonar mediante el abordaje de arteria y vena pulmonar y se objetiva sangrado dependiente de vena pulmonar derecha, inmediatamente se introducen los coils, consiguiendo embolización exitosa con 4 coils de $8 \times 5 \mathrm{~mm}$. Durante el procedimiento se pone de manifiesto la existencia de un gran derrame pleural derecho, se coloca un tubo torácico de $28 \mathrm{~F}$ que da salida a un hemotórax masivo de $3.800 \mathrm{cc}$.

La analítica en UCI mostraba Hto de $21 \%$, acidosis metabólica grave y parámetros de coagulopatía con actividad de protrombina de $17 \%$, ATTP > 2, fibrinógeno $90 \mathrm{mg} / 1$ y plaquetas 42.000 .

Se realiza tratamiento sustitutivo para corregir la coagulopatía. Inicialmente la paciente evoluciona bien consiguiendo estabilidad hemodinámica y normalizando parámetros de coagulación pero presenta fiebre de forma precoz con infección respiratoria que obliga a ventilación mecánica

La evolución posterior es desfavorable presentando bronconeumonía bilateral, aparición de fiebre y fallo renal, instaurandose un SDMO refractario. La paciente fallece tras 29 días de ingreso en UCI.

La perforación de la A. pulmonar es una complicación rara y a menudo fatal que puede producirse durante la cateterización de dicha arteria en cirugía cardiovascular, en la sala de hemodinámica o por el uso del catéter de Swan-Ganz. La incidencia de perforación varía por la bibliografía revisada pero parece oscilar según las series entre $0,1-1,5 \%$, condicionando una mortalidad de hasta el $50 \%$.

Los factores de riesgo y mecanismos involucrados $(1,2)$ en la patogénesis de este procedimiento son numerosos, destacando entre otros: la presencia de hipertensión pulmonar, sexo femenino, hipotermia, edad avanzada, valvulopatía mitral y la presencia de anticoagulación terapéutica.

Los signos clínicos de sospecha de perforación de la A. pulmonar varían desde una hemoptisis ligera a una gran hemorragia de la vía aerea con hipoxia, shock hipovolémico y parada cardiaca.

El tratamiento de la perforación de la arteria pulmonar incluye diagnóstico precoz mediante arteriografía, asegurar el intercambio gaseoso con lavado endobronquial, aislar el bronquio sangrante, ventilación mecánica y control de la hemorragia según la gravedad mediante tratamiento conservador, resección quirúrgica pulmonar y reparación de la arteria pulmonar o embolización arterial.

Actualmente cuál es el mejor tratamiento es controvertido, ya que el tratamiento conservador presenta una tasa alta de recurrencia de hemorragia y la cirugía no consigue reducir la mortalidad total. En este contexto la embolización se perfila cómo una alternativa segura especialmente en pacientes inestables, proporcionando un rápido control de la hemorragia (3). Las complicaciones descritas por esta técnica son escasas, que incluye la migración distal de los coils y el tamaño inadecuado para el vaso.

En aquellos pacientes con hemotórax, no obstante, continúa la recomendación (1) de realizar una toratocotomía para asegurar la supervivencia, realizando además una neumectomía, lobectomía o reparación vascular pulmonar sin demora.

En el caso que presentamos coexistían la mayoría de los factores de riesgo y aunque desconocemos cuál es el factor crítico que condiciono la rotura de la AP, sí reunía dos de los tres factores predisponentes (edad avanzada, existencia previa de HTP y anticoagulación). En otras series revisadas (5) la rotura de la AP se produce en la zona más distal a la estenosis seguramente favorecida por el hinchado del balón, restando importancia a la existencia de otros factores de riesgo.

La embolización de la AP en nuestra paciente fue rápida y eficaz en el control de la hemoptisis, si bien la gravedad del cuadro clínico y los signos de shock facilitaron los signos de encefalopa- tía con retraso en desconexiones de respirador y la mala evolución posterior.

No obstante, no queda claro si se debería haber planteado una cirugía torácica posterior a pesar de un buen control de la hemorragia pulmonar. La evacuación del hemotórax proporciono una buena reexpansión pulmonar y buen intercambio gaseoso, no existiendo datos que sugirieran la necesidad de una resección quirúrgica.

\section{B. Jiménez Bartolomé, J. González Cortijo, J. Medrano, E. Civeira Morillo ${ }^{1}$}

Servicios de Medicina Intensiva y ${ }^{\prime}$ Radiología Intevencionista. Hospital Clínico Universitario Lozano Blesa. Zaragoza

1. Tayoro J, Dequin PF, Delhommais A, Alison D, Perrotin D. Rupture of pulmonary artery induced by Swan-Ganz catheter: success of coil embolization. Intensive Care Med 1997; 23: 198-200.

2. Ivanov R, Allen J, Calvin JE. The incedence of major morbidity in critically ill patients managed with pulmonary artery catheters: a meta-analysis. Crit Care Med 2000; 28: No.3.

3. Sirivella S, Gielchinsky I, Parsonnet V. Management of catheter -induced pulmonary artery perforation: A rare complication in cardiovascular operations. Ann Thorac Surg 2001; 72: 2056-9.

4. Gottwalles Y, Wunschel-Joseph ME, Hanssen M. Coil Embolization treatment in pulmonary artery branch rupture during Swan-Ganz catheterisation. Cardiovasc Intervent Radiol 2000; 23: 477-9.

5. Baker CM, Mc Gowan FX Jr, Keane JF, Lock JE. Pulmonary artery trauma due to balloon dilation: recognition , avoidance and management. J Am Coll Cardiol 2000; 36: 1684-90.

6. Wunschel-Joseph ME, Gottwalles Y, Weisse D, Hanssen M .Rupture d'une branche de l'artère pulmonaire au cours d'un cathétérisme de Swan-Ganz. Archives des maladies du cœur et des vaisseaux, tome $92 \mathrm{n}^{\circ}$ 2 février 1999.

\section{Obstrucción parcial de intestino delgado en paciente con enfermedad de von Recklinghausen}

\section{Sr. Director:}

La neurofibromatosis (NF) tipo 1 o enfermedad de von Recklinghausen es un trastorno mucocutáneo caracterizado por la presencia de neurofibromas múltiples y lesiones pigmentadas de la piel (manchas café con leche). Puede asociarse con otros tumores del sistema nervioso central, lesiones lentiginosas de la axilas, hamartomas pigmentarios del iris, feocromocitoma y en ocasiones con trastornos convulsivos y retraso mental.

El tracto gastrointestinal se encuentra afectado en el 11-25\% de los casos (1-3). En la mayoría de los pacientes la afectación suele ser asintomática. En raras ocasiones, puede cursar con dismotilidad de intestino delgado y pseudoobstrucción intestinal (4). Presentamos un caso de obstrucción parcial de intestino delgado en un paciente con enfermedad de von Recklinghausen.

Se trata de un varón de 65 años, con neurofibromatosis de von Recklinghausen e hipertrofia benigna de próstata en tratamiento con alfuzosina y tamsulosina clorhidrato. El paciente ingresa por un cuadro de dos meses de evolución de dolor abdominal recurrente en hemiabdomen inferior e hipogastrio, que mejoraba con la defecación y tras analgesia con metamizol. No se asociaba a náuseas, vómitos, fiebre ni rectorragia. En la exploración física 
destacaban neurofibromas múltiples generalizados mas prominentes en tórax y espalda. Presentaba un abdomen blando, depresible, levemente doloroso a la palpación en hemiabdomen inferior, sin signos de irritación peritoneal, con ruidos hidroaéreos aumentados. En el resto de la exploración, incluida la neurológica, no presentaba alteraciones de interés. En el hemograma encontramos 10.300 miles $/ \mu \mathrm{L}$, leucocitos con $83 \%$ de granulocitos. En la bioquímica proteínas totales de 5,6 g/dl con 2,9 g/dl de albúmina y $13 \%$ de gammaglobulinas. El resto de los parámetros, incluidos los reactantes de fase aguda y marcadores tumorales CEA y CA 19.9 presentaban valores dentro de los rangos de la normalidad. Ante la persistencia de síntomas y sospecha de patología a nivel colónico, se realizó una colonoscopia donde se objetivó a nivel del orificio de entrada de la válvula ileocecal una mucosa de aspecto velloso, friable, erosionada que condicionaba estenosis con imposibilidad de pasar a íleon terminal. Se tomaron biopsias para estudio anatomopatológico informadas como erosión mucosa y proceso inflamatorio inespecífico. No se hallaron datos compatibles con neoplasia, isquemia ni proceso infeccioso. La tinción de Zielh-Neelsen y el cultivo para mycobacterias fueron negativos. El resto de la mucosa colónica hasta ciego no presentaba alteraciones. Para completar el estudio se realizó una tomografía axial computerizada abdomino-pélvica que no mostró alteraciones de interés, y un tránsito intestinal en el que se objetivó intestino delgado con múltiples imágenes de defectos de repleción en relación con formaciones polipoideas, que no provocaban obstrucción destacaba una estenosis a nivel de íleon terminal filiforme fija, sin poder aportar mas datos sobre su etiología. Dada la persistencia de cuadros de dolor abdominal recurrente durante el ingreso y el hallazgo de la estenosis de íleon terminal de etiología no filiada, se decidió realizar laparotomía con intención diagnóstico-terapéutica. Macroscópicamente se observaron múltiples lesiones polipoideas serosas en estómago y yeyuno proximal compatibles con neurofibromas, con poliposis difusa intestinal. Junto a esto, una gran masa en el mesenterio del íleon terminal retráctil, cuya biopsia intraoperatoria mostró linfadenitis reactiva con fibrosis periganglionar. Se realizó resección ileocólica segmentaria incluyendo $30 \mathrm{~cm}$ de íleon terminal y ciego con anastomosis ileocólica. El informe anatomopatológico de la pieza resecada en intestino delgado se informó como hiperplasia y proliferación de células ganglionares y fibras nerviosas en submucosa y lámina propia compatible con ganglioneuromatosis intestinal difusa, junto a pólipos inflamatorios y formación polipoidea en la válvula ielocecal de $0,8 \mathrm{~cm}$ que correspondía a neurofibroma. Tras la intervención el paciente evolucionó favorablemente, ha permanecido asintomático en posteriores revisiones.

La neurofibromatosis tipo 1 o enfermedad de von Recklinhgausen afecta el tracto gastrointestinal en un 11-25\% de los casos, siendo los tramos más frecuentemente afectados el estómago y yeyuno. Las lesiones descritas a estos niveles son neurofibromas, ganglioneuromas y swchannomas (1-3).

Aunque la afectación digestiva suele ser asintomática y descubrirse de forma incidental, puede originar cuadros clínicos que requieren cirugía. Las formas de presentación más habituales son la hemorragia y el dolor abdominal secundario a obstrucción. Otras formas menos frecuentes son la dismotilidad de intestino delgado y la pseudoobstrucción intestinal en relación con afectación de los plexos mientéricos, pudiendo originar incluso un megacolon o angina abdominal por compresión arterial (5). Desde el punto de vista histológico se caracteriza por hiperplasia de células ganglionares y fibras nerviosas ente ambas capas musculares y lámina propia, en la que se encuentran neuronas deformadas con axones desorganizados (2).

En el caso referido, la afectación digestiva de la enfermedad de von Reklinghausen se manifestó con episodios de dolor abdominal recurrente en relación con cuadros suboclusivos intestinales, se descartaron las causas más frecuentes relacionadas con la enfermedad de base, como la obstrucción por intususcepción y las altera- ciones de la motilidad. En las técnicas de imagen, incluido el tránsito intestinal y la colonoscopia, se identificó una estenosis fija en íleon terminal sin poder precisar la etiología por la inespecificidad de las biopsias obtenidas. Ante la duda diagnóstica de afectación inflamatoria, neoplásica o isquémica de íleon terminal se procedió a laparotomía que permitió el diagnóstico definitivo, mostró la existencia de una estenosis asociada a masa mesentérica ileal secundaria a linfadenitis reactiva con fibrosis periganglionar compatible con lo descrito en la literatura como tumor denso o neurofibromatosis plexiforme (5). El estudio completo de la pieza quirúrgica permitió descartar de forma definitiva la existencia de patología neoplásica o infecciosa sobreañadida. La mayoría de las complicaciones gastrointestinales de la enfermedad de von Recklinghausen requieren cirugía para su resolución, como sucedió en nuestro caso. Tras revisar la literatura, consideramos de interés la comunicación de este caso dada la rareza de esta forma de presentación gastrointestinal de la enfermedad.

\section{D. Mañas, B. Piqueras ${ }^{1}$, E. Marchán, A. Hernández ${ }^{1}$}

Servicio de Medicina Interna. ${ }^{I}$ Sección Aparato Digestivo. Complejo Hospitalario de Ciudad Real. Ciudad Real

1. Martínez-Ordaz JL, Esmer-Sánchez DD, Suárez-Moreno R, Ruiz-Roque $\mathrm{R}$, Blanco-Benavides R. Gastrointestinal neurofibromatosis. A rare cause of abdominal surgery. Rev Gastroenterol Mex 2000; 65: 34-36.

2. García Aroca J, Sanz N, Alonso JL, de Mingo L, Rollan V. Intestinal pseudo-obstruction secondary to systemic neuropathies and myopathies. Cir Pediatr 1994; 7: 115-120.

3. Artaza T, García JF, González C, Amengual M, Mazarro A, Rodríguez $\mathrm{R}$, et al. Simultaneous involvement of the jejunum and the colon by type1 neurofibromatosis. Scand J Gastroenterol 1999; 34: 331-334.

4. Sinha SK, Kkochhar R, Rana S, Bapuraj S, Singh K. Intestinal pseudoobstruction due to neurofibromatosis responding to cisapride. Indian J Gastroenterol 2000; 19: 83-84.

5. Phat VN, Sezeur A, Danne M, Dupuis D, de la Vaissiere G, Camilleri JP. Primary myenteric plexus alteration as a cause of megacolon in Von Recklinhausen's diseases. Pathol Biol (Paris) 1980; 28: 585-588.

\section{Hemobilia como complicación de biopsia hepática transyugular}

\section{Sr. Director:}

La hemobilia se define como una comunicación patológica entre los conductos biliares y los vasos sanguíneos. Clínicamente se manifiesta como un cólico biliar con ictericia y hemorragia digestiva, y es causada en la mayor parte de los casos por traumatismos hepáticos (1). Se ha descrito como complicación infrecuente de la biopsia hepática percutánea y, excepcionalmente, tras la realización de la biopsia hepática transyugular (2). Por este motivo consideramos de especial interés la comunicación del siguiente caso clínico.

Varón de 70 años que ingresa por dolor en hipocondrio derecho de un mes de evolución. En los análisis destacaba elevación de enzimas hepáticas (GOT 76 UI/L, GPT 104 UI/L, GGT 362 UI/L, FA 770 UI/L, LDH 365 UI/L), anemia normocítica normocrómica (Hb 9.7 g/dL) y reactantes de fase aguda (VSG $109 \mathrm{~mm}$ en la $1^{\text {a }}$ hora, proteína $\mathrm{C}$ reactiva $182 \mathrm{mg} / \mathrm{L}$, fibrinógeno 782 $\mathrm{mg} / \mathrm{dL}$ ) con el resto de los parámetros bioquímicos incluido bilirrubina dentro de la normalidad. Para el estudio del cuadro que 
motivó su ingreso se realizaron ecografía y tomografía computerizada (TC) abdominal que evidenciaron una hepatomegalia con aumento de ecogenicidad, y análisis con autoanticuerpos y serologías para virus de la hepatitis B y C, cuyos resultados fueron normales o negativos. Durante su ingreso presentó un cuadro de dolor pleurítico en costado derecho y expectoración hemoptoica, siendo diagnosticado mediante pruebas de imagen de tromboembolismo pulmonar y trombosis venosa profunda, por lo que se inició anticoagulación con heparinas de bajo peso molecular (HBPM).

Ante la inespecificidad de las pruebas realizadas, y debido a la persistencia de la sintomatología y de las alteraciones analíticas concordantes con colestasis disociada, se decidió la realización de biopsia hepática mediante vía transyugular, para la cual se suspendió la anticoagulación el día previo y se reinició el día posterior al procedimiento, con enoxaparina $1 \mathrm{mg} / \mathrm{kg} / 24$ horas. Cinco días después del procedimiento comenzó con dolor abdominal brusco en hipocondrio derecho y anemización con melenas que precisó transfusión de dos concentrados de hematíes. La TC abdominal con contraste no mostró alteraciones, por lo que se realizó gastroscopia donde se objetivó sangrado activo a través del esfínter coledocoduodenal. Con el diagnóstico de hemobilia se instauró dieta absoluta y el paciente se mantuvo estable, reiniciándose el tratamiento con HBPM a dosis profilácticas cuatro días después. Tras 15 días, y estando ya asintomático, desarrolló de nuevo melenas con anemización. Debido a la falta de respuesta del tratamiento médico se procedió a la realización de arteriografía que visualizó el punto de sangrado y se embolizó hasta su completa oclusión. El estudio histopatológico del hígado fue informado como cambios inespecíficos. Posteriormente no ha desarrollado nuevas complicaciones apreciándose normalización de las enzimas hepáticas.

La hemobilia, entidad descrita por primera vez en 1654 por Glisson, puede ver aumentada su incidencia en los próximos años debido al incremento en el número de técnicas invasivas a nivel de hígado y vías biliares que se realiza, y a la actitud actual más conservadora de los traumatismos hepáticos accidentales (3). Su aparición como complicación de la biopsia hepática transyugular como en nuestro caso es infrecuente, si bien es de reseñar que este paciente estaba anticoagulado, lo que sin duda favoreció la hemorragia recidivante.

El sangrado que se produce puede variar en cuantía y ocurrir a cualquier nivel del tracto biliar, aunque se localiza más frecuente- mente en las vías biliares intrahepáticas y vesícula biliar (1). La presentación clínica tras el traumatismo tiene una duración variable, y depende de la cuantía del sangrado, la necrosis hepática, la formación de un pseudoaneurisma y la presencia de bilis, pues ésta inhibe la formación de fibrina y la granulación (3). Además, pueden producirse como complicaciones colecistitis, colangitis y pancreatitis (4).

El diagnóstico de hemobilia en ocasiones es difícil de realizar, ya que tanto la ecografía y la TC, como la endoscopia y la colangiopancreatografía retrógrada endoscópica, tienen una baja sensibilidad, y en ocasiones es necesario recurrir a la arteriografía para el diagnóstico de esta entidad, con la ventaja añadida terapéutica que posee esta técnica (3).

Habitualmente suele producirse una resolución espontánea, aunque en ocasiones precisa la realización de procedimientos terapéuticos invasivos. Entre las distintas opciones se encuentran la hepatectomía parcial, la ligadura de las arterias hepáticas y la embolización arterial, técnica que utilizamos en nuestro paciente. Esta última ha ganado una amplia aceptación en lugar del tratamiento quirúrgico debido a su menor morbimortalidad.

Debemos tener en cuenta la hemobilia como causa de hemorragia digestiva, fundamentalmente cuando se asocia a dolor abdominal y consta como antecedente la existencia de un traumatismo o la realización de cualquier procedimiento invasivo hepático previo.

\section{P. Álvarez Padín, F. J. Fernández Fernández, C. Martínez Vázquez, S. Vázquez López, A. Corbillón Estévez}

Servicio de Medicina Interna. Complexo Hospitalario Universitario Xeral-Cíes. Universidad de Santiago de Compostela. Vigo

1. Sandblom P, Saegesser F, Mirkovitch V. Hepatic hemobilia: hemorrhage from the intrahepatic biliary tract, a review. World J Surg 1984; 8: 41-50.

2. Gorriz E, Reyes R, Lobrano MB, Pulido-Duque JM, San Román JL, Lonjedo E, Ferral H, Maynar M. Transjugular liver biopsy: a review of 77 biopsies using a spring-propelled cutting needle (biopsy gun). Cardiovasc Intervent Radiol 1996; 19: 442-445.

3. Green MHA, Duell RM, Johnson CD, Jamieson NV. Haemobilia. Br J Surg 2001; 88: 773-786.

4. Van Os EC, Petersen BT. Pancreatitis secondary to percutaneous liver biopsy-associated hemobilia. Am J Gastroenterol 1996; 91: 577-580. 